\title{
Regulation of pseudo-Polyrotaxane Formation between $\alpha$-Cyclodextrins and Azobenzene-Terminated Poly(ethylene glycol)
}

\author{
Taichi IKeDa, Tooru Ooya, and Nobuhiko YUI ${ }^{\dagger}$ \\ School of Materials Science, Japan Advanced Institute of Science and Technology, \\ 1-I Asahidai, Tatsunokuchi, Ishikawa 923-1292, Japan
}

(Received January 19, 1999)

\begin{abstract}
Regulation of pseudo-polyrotaxane formation between $\alpha$-cyclodextrins ( $\alpha$-CDs) and azobenzene(Az)terminated poly(ethylene glycol) (PEG) was studied in terms of the photoisomerization of the terminal Az groups. pseudo-Polyrotaxane formation was analyzed by a change in the transmittance at $550 \mathrm{~nm}$ in water. It was found that the rate of transmittance change was decreased with an increase in the cis isomer content in Az-PEG. The cis-isomerization effect was quantitatively evaluated, assuming that Az-PEG having two cis-isomerized Az groups does not participate in the pseudo-polyrotaxane formation. From this analysis, it is suggested that pseudo-polyrotaxane formation between $\alpha$-CDs and PEG was hindered when the both terminal Az groups were isomerized to cis isomer. The content of Az-PEG in the pseudo-polyrotaxane aggregate and the content of cis-isomerized Az-PEG in the supernatant were measured by ${ }^{1} \mathrm{H}$ NMR and UV-Vis absorption spectrum, respectively, and the obtained results supported our suggestion. It is concluded that the terminal $\mathrm{Az}$ groups act as a gate to regulate the pseudo-polyrotaxane formation.

KEY WORDS pseudo-Polyrotaxane Formation / Photoisomerization / $\alpha$-Cyclodextrin / Poly(ethylene glycol) / Azobenzene /
\end{abstract}

Over the past two decades, new synthetic methods have been investigated in order to design "intelligent" or "smart" materials which exhibit large property change in response to small physical or chemical stimuli. ${ }^{1}$ In particular, the progress in supramolecular chemistry has introduced a new fascinating field of nano-organized systems mimicking nanostructures in nature. ${ }^{2,3}$ The nano-organized systems have been focused on a key technology to realize high-performance smart materials, micromachines and so on. Molecular assemblies organized by their intermolecular forces are believed to be essential for the construction of the nano-organized systems. $^{4}$

pseudo-Polyrotaxanes in which many cyclic molecules are threaded onto a linear polymer have been recently studied as a novel molecular assembly. ${ }^{5-8}$ Harada et al. demonstrated pseudo-polyrotaxane formation between cyclodextrins (CDs) and a linear polymer in high yield. It was found that $\alpha-, \beta$-, or $\gamma$-CDs were selectively threaded onto various linear polymers. ${ }^{9}$ For instance, when $\alpha$-CD saturated aqueous solution is mixed with poly(ethylene glycol) (PEG) aqueous solution, the solutions become turbid. ${ }^{10}$ This phenomenon is caused by the formation of pseudo-polyrotaxane and its aggregation. Further, when bulky-terminal groups are introduced to the terminals of the polymer, the sterical hindrance for pseudo-polyrotaxane formation is changed in relation to the shape of the bulky-terminal groups. For instance, Harada et al. reported that bis(2naphthylacetyl)-terminated PEG forms a pseudo-polyrotaxane with $\alpha-C D$, whereas bis(1-naphthylacetyl)terminated PEG does not. ${ }^{11}$ This report indicates that the pseudo-polyrotaxane formation is affected by the mobility or shape of the terminals of the linear polymer. If stimuli-responsible moieties, which can control the mobility or shape by external stimuli, are introduced to the terminals of PEG chain, the threading of many $\alpha$-CDs onto the PEG chain may be regulated by physicochemical stimuli such as light, $\mathrm{pH}$, and temperature. Since pseudo-polyrotaxane formation is accompanied by a change in the physicochemical properties of the solution such as viscosity, ${ }^{12,13}$ its regulation by external stimuli may switch the physicochemical properties of the solution. Azobenzene (Az) is a well-known photosensitive molecule which transforms from trans isomer to cis isomer upon UV irradiation. Since its isomerization is accompanied by a change in the physical and chemical properties such as polarity and molecular shape, ${ }^{14}$ it is possible to regulate inclusion complexation between $\mathrm{Az}$ and $\alpha-C D$. Using the photoisomelism characteristics of Az, Nakashima et al. have demonstrated a light-driven molecular shuttle utilizing $\alpha-C D$ and an azobenzenecontaining polymer capped with 1,4-dinitrobenzene. ${ }^{15}$ They clarified that $\mathrm{Az}$ moiety is captured in the cavity of $\alpha-C D$ when $\mathrm{Az}$ forms trans isomer, and that $\alpha-\mathrm{CD}$ exists on the polymer chain beside $\mathrm{Az}$ moiety when $\mathrm{Az}$ forms $c i s$ isomer. This report indicates that a change in the geometrical shape of $\mathrm{Az}$ plays an important role to regulate the position of $\alpha-C D$ on the Az-containing polymer. Namely, the interaction between $\mathrm{Az}$ and $\alpha-\mathrm{CD}$ cavity is likely to be sterically hindered when $\mathrm{Az}$ forms cis isomer. Thus, $\mathrm{Az}$ is considered to be suitable for a model of stimuli-responsible terminal groups to regulate the pseudo-polyrotaxane formation.

In this paper, pseudo-polyrotaxane formation between $\alpha$-CDs and azobenzene-terminated PEG (Az-PEG) was studied in relation to the photoisomerization. The aggregation behavior in relation to the pseudo-polyrotaxane formation was analyzed by the rate of transmittance change and its dependence on the cis isomer content of Az-PEG was discussed. Furthermore, the effect of the isomerization of the terminal-Az groups on

\footnotetext{
† To whom correspondence should be addressed (Tel: +81-761-51-1640, Fax: +81-761-51-1645, e-mail: yui@jaist.ac.jp).
} 

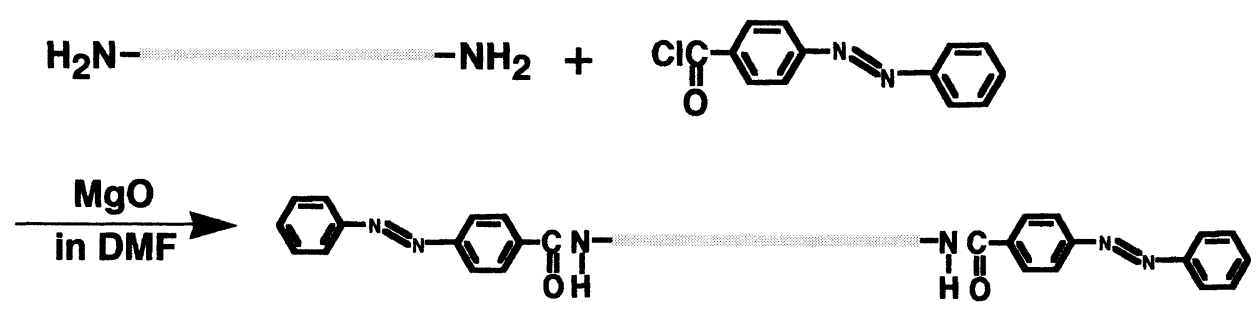

$$
\left(=\left(\mathrm{CH}_{2}\right)_{3}\left(\mathrm{OCH}_{2} \mathrm{CH}_{2}\right)_{\mathrm{a}} \mathrm{O}\left(\mathrm{CH}_{2}\right)_{3}, \mathrm{a}=91\right)
$$

Scheme 1. Preparation of azobenzene-terminated PEG.

the pseudo-polyrotaxane formation was evaluated by the content of Az-PEG in the pseudo-polyrotaxane aggregate $\left(\{\mathrm{Az}-\mathrm{PEG}\}_{\text {prx }}\right)$ and the content of cis-isomerized Az-PEG in the supernatant after centrifugation of the pseudo-polyrotaxanes aggregate.

\section{EXPERIMENTAL}

\section{Materials}

$\alpha$-Cyclodextrin ( $\alpha$-CD), $p$-phenylazobenzoylchloride $(\mathrm{AzCOCl})$ and magnesium oxide $(\mathrm{MgO})$ were purchased from Wako Pure Chemical Co., Ltd. (Osaka, Japan). $\alpha$-(3-Aminopropyl)- $\omega$-(3-aminopropyl)polyoxyethylene with $\bar{M}_{n}$ of 4000 (PEG-BA) was kindly supplied by Sanyo Chemical Co., Ltd. (Kyoto, Japan) as IONET ${ }^{\circledR}$ YB-400. Solvents were purified as usual.

\section{Preparation of $A z-P E G$}

Az-PEG was prepared from PEG-BA according to the similar method described by Gallot et al. (Sheme 1). ${ }^{16}$ PEG-BA $(0.21 \mathrm{~g} ; 0.05 \mathrm{mmol})$ was dissolved in $N, N$ dimethylformamide (DMF) $(20 \mathrm{~mL}) . \mathrm{AzCOCl}(0.25 \mathrm{~g}$; $1.0 \mathrm{mmol}$ ) and excess amount of $\mathrm{MgO}$ were added to the PEG-BA solution, and then the mixture was stirred under a nitrogen atmosphere for $24 \mathrm{~h}$ at room temperature. The product was precipitated from ether to remove unreacted $\mathrm{AzCOCl}$. The Az-PEG aqueous solution was dialized against distilled water to remove $\mathrm{MgO}$. The resulting solution was freeze-dried to obtain a crude product as a yellow powder. Finally, the product was purified by gel permeation chromatography (GPC) using Sephadex LH-20 (fine) with methanol as an eluent. Total yield: $80 \%$. ${ }^{1} \mathrm{H}$ NMR (dimethyl sulfoxide (DMSO)- $d_{6}$ ): $\delta$ $7.60-8.45\left(\mathrm{~m}\right.$, aromatic of azobenzene), $\delta 3.50\left(\mathrm{~s}, \mathrm{CH}_{2}\right.$ of PEG); IR (KBr): 1637 (amide $\mathrm{C}=\mathrm{O}), 1564(\mathrm{~N}-\mathrm{H})$ $\mathrm{cm}^{-1} ; \mathrm{UV}_{\max }\left(\right.$ in $\left.\mathrm{H}_{2} \mathrm{O}\right) 325 \mathrm{~nm}$.

The introduction of $\mathrm{Az}$ at the terminals of PEG was confirmed by HPLC equipped with a UV detector (UV-970, Jasco, Tokyo, Japan) and a refractive index detector (RI-930, Jasco, Tokyo, Japan) by using a Shodex GF-510HQ column [300 7.6 (i.d.) $\mathrm{mm}]$ (Showa Denko Co., Ltd., Tokyo, Japan) and DMF as an eluent at $30^{\circ} \mathrm{C}$. The functionality of $\mathrm{Az}$ groups in PEG was determined by ${ }^{1} \mathrm{H}$ NMR and UV absorption spectrum. From the ${ }^{1} \mathrm{H}$ NMR spectrum using a $300 \mathrm{MHz}$ FT-NMR (Gemini300, Varian, Palo Alto, CA, U.S.A.) in DMSO$d_{6}$, the functionality was calculated from the integral of the aromatic peaks of $\mathrm{Az}$ and that of the methylene protons on PEG. From the UV absorption spectrum using a UV-Vis spectrophotometer (V-550, Jasco, Tokyo,
Table I. Preparation and characterization of pseudo-polyrotaxane formation between $\alpha-\mathrm{CD}$ and $\mathrm{Az}-\mathrm{PEG}$

\begin{tabular}{cccccc}
\hline Code & $\begin{array}{c}{[\mathrm{Az}-\mathrm{PEG}]^{\mathrm{a}}} \\
\times 10^{4} \mathrm{M}\end{array}$ & $\{c i s\}^{\mathrm{b}}$ & $C_{\mathrm{RX}} \mathrm{c}^{\mathrm{c}}$ & $\begin{array}{c}{[\mathrm{Az}-\mathrm{PEG}]_{\mathrm{Rx}}{ }^{\mathrm{d}}} \\
\times 10^{4} \mathrm{M}\end{array}$ & $\begin{array}{c}\mathrm{r}^{\mathrm{e}} \\
\times 10^{2} \mathrm{~min}^{-1}\end{array}$ \\
\hline Az-PEG1 & 1.52 & 0.00 & 1.00 & 1.52 & 1.41 \\
Az-PEG2 & 1.27 & 0.00 & 1.00 & 1.27 & 1.18 \\
Az-PEG3 & 1.14 & 0.00 & 1.00 & 1.41 & 1.03 \\
Az-PEG4 & 1.52 & 0.29 & 0.96 & 1.46 & 1.35 \\
Az-PEG5 & 1.52 & 0.64 & 0.83 & 1.26 & 1.19 \\
\hline
\end{tabular}

${ }^{\mathrm{a}} \mathrm{Az}-\mathrm{PEG}$ total concentration. ${ }^{\mathrm{b}}$ The content of cis isomer determined by UV absorption spectrum. ${ }^{\mathrm{c}}$ The content of the 1,0-cisAz-PEG. ${ }^{\mathrm{d}}$ Concentration of Az-PEG participating in the pseudo-polyrotaxane formation. ${ }^{\mathrm{e}}$ The rate of transmittance change.

Japan), the concentration of Az groups in the solution was determined in comparison with the absorbance of low molecular weight model compound, $p$-phenylazobenzoyl-glycine (Az-Gly) $\left(\varepsilon_{\text {trans }}=24000 \mathrm{~L} \mathrm{~mol}^{-1} \mathrm{~cm}^{-1}\right.$ and $\varepsilon_{c i s}=1500 \mathrm{~L} \mathrm{~mol}^{-1} \mathrm{~cm}^{-1}$ at $325 \mathrm{~nm}$ in water), which was synthesized according to the similar method by Fissi et al. ${ }^{17}$ The first-order kinetic constant of cis $\rightarrow$ trans thermal isomerization $(k)$ was determined by the measurement of time course change in absorbance at $325 \mathrm{~nm}$ using the UV-Vis spectrophotometer $(k=4.8 \times$ $\left.10^{-6} \mathrm{~s}^{-1}\right)$.

\section{Transmittance Measurements}

The Az-PEG aqueous solution $\left(1.52 \times 10^{-4} \mathrm{M}\right)$ was prepared as a stock solution. Then the solutions of Az-PEG1-3 in Table I were prepared from the stock solution by dilution. Photoisomerization of the Az-PEG4 and 5 solutions $\left(1.52 \times 10^{-4} \mathrm{M}\right)$ were carried out by UV-irradiation at $330 \mathrm{~nm}$ using a 500-W high-pressure mercury lamp (BMO-500D1, M. Watanabe \& Co., Ltd., Tokyo, Japan) with a band-pass filter (UV-D33S, Toshiba, Tokyo, Japan) for $2 \mathrm{~min}$ (Az-PEG4) and $1 \mathrm{~h}$ (Az-PEG5). $\alpha$-CD saturated solution $(2.0 \mathrm{~mL}, 1.50 \times$ $\left.10^{-1} \mathrm{M}\right)$ in a UV cell was set in the UV-Vis spectrophotometer with stirring, and then each Az-PEG aqueous solution $(1.0 \mathrm{~mL})$ was added to the solution. The transmittance at $550 \mathrm{~nm}$ was measured at $25^{\circ} \mathrm{C}$. The content of cis-isomerized Az at the PEG terminals ( $\{c i s\}$ in Table I) was determined by the absorbance at $325 \mathrm{~nm}$ in comparison with that of Az-Gly.

\section{Calculation of the Rate of Transmittance Change}

The plot of transmittance $v s$. time was used for calculating the rate of transmittance change. The slope of 
Table II. Characterization of pseudo-polyrotaxane formation between $\alpha$-CD and Az-PEG

\begin{tabular}{|c|c|c|c|c|}
\hline Code & $\left\{(c i s\}^{\mathrm{a}}\right.$ & $\begin{array}{c}\{\mathrm{Az}-\mathrm{PEG}\}_{\text {feed }}^{\mathrm{b}} \\
\quad \times 10^{6} \mathrm{~mol}\end{array}$ & $\begin{array}{l}\{\mathrm{Az}-\mathrm{PEG}\}_{\mathrm{prx}}^{\mathrm{c}} \\
\quad \times 10^{6} \mathrm{~mol}\end{array}$ & \\
\hline Az-PEG6 & 0.00 & 1.14 & $1.08 \pm 0.02$ & \multirow{3}{*}{ ** } \\
\hline Az-PEG7 & 0.33 & 1.14 & $1.06 \pm 0.02$ & \\
\hline Az-PEG8 & 0.54 & 1.14 & $1.02 \pm 0.02$ & \\
\hline
\end{tabular}

a The content of $c$ is isomer determined by UV absorption spectrum $\varepsilon_{\text {truns }}=2400 ; \varepsilon_{\text {cis }}=1500 .{ }^{\mathrm{b}}$ Concentration of Az-PEG in feed on the preparation of the pseudo-polyrotaxane. ${ }^{\mathrm{c}}$ Content of the Az-PEG in the precipitated pseudo-polyrotaxane; $*$ and $* *$ were significantly different at $p<0.1$ and $p<0.05$, respectively, calculated using $t$-test.

the transmittance curve in a linear region was defined as the rate of transmittance change. The values of the rate were obtained by least-squares fit in the linear region.

\section{Preparation of pseudo-Polyrotaxanes Consisting of $\alpha-C D$ and $A z-P E G$}

The Az-PEG aqueous solution $\left(5.7 \times 10^{-3} \mathrm{M}\right)$ was prepared as a stock solution. Photoisomerization of the Az-PEG7 and 8 solutions in Table II was carried out by UV-irradiation at $330 \mathrm{~nm}$ using a $500-\mathrm{W}$ high-pressure mercury lamp with a band-pass filter for $50 \mathrm{~min}(\mathrm{Az}-$ PEG7) and $3 \mathrm{~h}$ (Az-PEG8). pseudo-Polyrotaxanes were prepared from $\alpha-C D$ and the Az-PEGs (Table II ), according to the method previously reported by Harada et al. ${ }^{10} \mathrm{Az}-\mathrm{PEG}$ aqueous solution $(0.2 \mathrm{~mL})$ was added to the $\alpha-C D$ saturated solution $\left(2.0 \mathrm{~mL}, 1.50 \times 10^{-1} \mathrm{M}\right)$ in a centrifuge tube. The mixtures were ultrasonically agitated for a few minutes and then allowed to stand for $1 \mathrm{~h}$ at room temperature. The precipitated products were separated by ultracentrifugation $(15000 \mathrm{rpm} \times 15 \mathrm{~min})$. After the decantation to remove the supernatant, the yellow solid products were dried under vacuum at $40^{\circ} \mathrm{C}$ to give a pseudo-polyrotaxane. From a ${ }^{1} \mathrm{H}$ NMR spectrum in DMSO- $d_{6}$, the content of Az-PEG in the collected pseudo-polyrotaxane $\left(\{\mathrm{Az}-\mathrm{PEG}\}_{\mathrm{prx}}\right)$ was calculated from the integral of $\mathrm{H}-1$ protons of $\alpha-\mathrm{CD}$ and that of the methylene protons on PEG. In order to characterize the Az-PEG in supernatant, UV-Vis absorption spectrum of the supernatant after centrifugation was measured using the UV-Vis spectrophotometer.

\section{RESULTS AND DISCUSSION}

\section{Characterization of $A z-P E G$}

Figure 1 shows the elution diagram of Az-PEG after the purification. Az-PEG was detected by the absorbance at $440 \mathrm{~nm}$ at elution volume of $8.5 \mathrm{~mL}$ ((a) in Figure 1). Az-PEG was also detected by the refractive index (data not shown). A peak of the elution volume at $5 \mathrm{~mL}$ (void volume) may be due to an association of Az-PEG in DMF. PEG-BA was detected at similar elution volume $(8.5 \mathrm{~mL})$ by refractive index ((b) in Figure 1$)$, although it was not detected by the absorbance at $440 \mathrm{~nm}$ (data not shown). AzCOCl was detected at a lower molecular weight position than that of Az-PEG ((c) in Figure 1). Furthermore, amide bond between PEG and $\mathrm{Az}$ was confirmed by the IR measurement, and aromatic protons of $\mathrm{Az}$ and methylene protons on PEG were also confirmed by the ${ }^{1} \mathrm{H}$ NMR spectrum (see EXPERI-

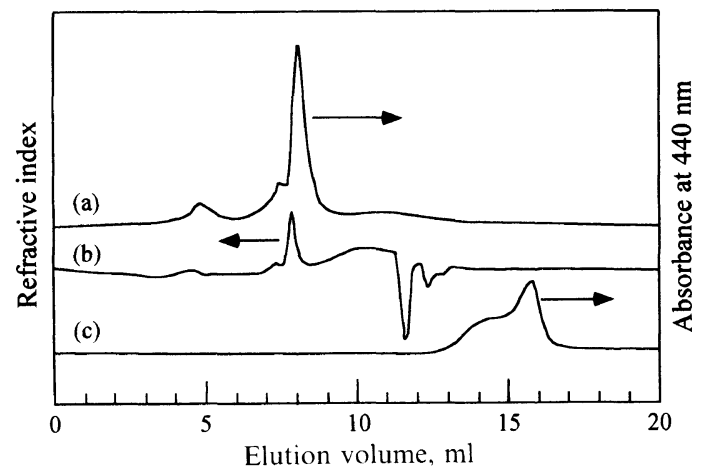

Figure 1. Elution diagram of Az-PEG (a), PEG-BA (b), and AzCOCl (c) (column, Shodex GF-510HQ $(300 \times 7.6$ (i.d.) $\mathrm{mm})$; eluent, DMF; flow rate, $0.5 \mathrm{~mL} \mathrm{~min}^{-1}$ ). Absorbance was measured at $\lambda=440 \mathrm{~nm}$.

MENTAL). These results indicate that $\mathrm{Az}$ is actually introduced at the terminal of PEG and that unreacted $\mathrm{Az}$ is completely removed. The functionality of Az groups in PEG was found to be $c a .1 .3$ by the ${ }^{1} \mathrm{H}$ NMR spectrum and UV absorption spectrum. In this study, the effect of the cis-isomerization of Az-PEG on the pseudopolyrotaxane formation was analyzed by the rate of transmittance change. The Az-PEG fraction which has only one $\mathrm{Az}$ moiety should participate in the pseudopolyrotaxane formation even photoisomerization occurs, because at least the one-end is always free. Thus the change in the pseudo-polyrotaxane formation may be based on the cis-isomerization of bifunctional Az-PEG. The content of bifunctional Az-PEG in the Az-PEG solution is estimated to be $0.42\left((1.3 / 2)^{2}\right)$. Though this content of bifunctional Az-PEG is not desirable to the perfect regulation of the pseudopolyrotaxane formation, it is considered to be enough high to confirm the effect of cis-isomerization on the pseudo-polyrotaxane formation.

\section{The Rate of Transmittance Change: Its Dependence on the Az-PEG Concentration}

When PEG aqueous solution is added to $\alpha-C D$ saturated aqueous solution, the mixed solution becomes turbid. ${ }^{10}$ This phenomenon is considered to involve two processes; one is the threading process (pseudo-polyrotaxane formation), and the other is the aggregation process of the pseudo-polyrotaxane. ${ }^{18}$ In order to confirm the aggregation process accompanied by the pseudopolyrotaxane formation between Az-PEG and $\alpha-C D$ in water and to obtain the relationship between the Az-PEG concentration and the pseudo-polyrotaxane formation, transmittance change caused by the aggregation of the pseudo-polyrotaxane was measured using all-trans isomerized Az-PEG (Az-PEG1-3 in Table I) . It is noted that the rate of transmittance change depends on the molecular weight of PEG, which has already been reported by Harada et al. ${ }^{10}$ It was confirmed that the molecular weight distribution of Az-PEG did not change before and after pseudo-polyrotaxane formation by GPC. Then, the influence of the polydispersity in the rate of the transmittance change may be negligible.

Figure 2 shows transmittance curves when each AzPEG1 -3 aqueous solution was added to the $\alpha-C D$ saturated solution. The decrease in transmittance indicates that the trans-isomerized Az-PEG forms a pseudo-poly- 


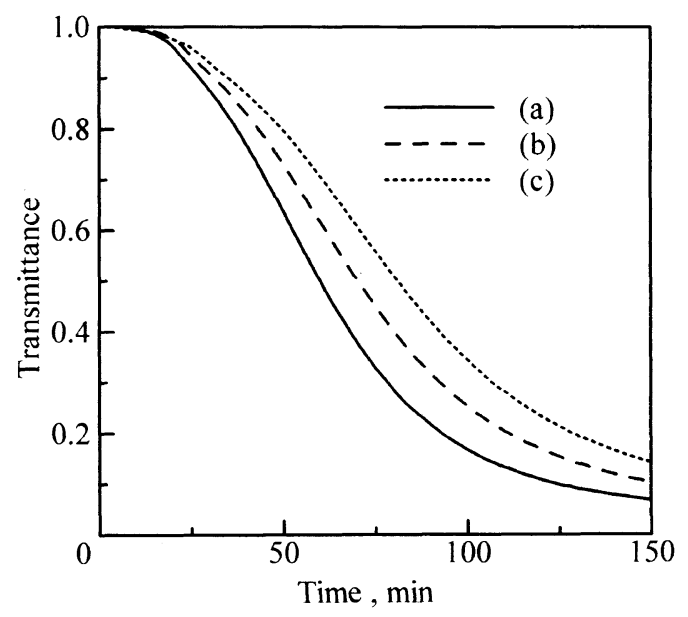

Figure 2. Transmittance curve at $550 \mathrm{~nm}$ for pseudo-polyrotaxane formation between $\alpha-C D$ and Az-PEG1 (a), Az-PEG2 (b), and Az-PEG3 (c) in water at $25^{\circ} \mathrm{C}$.

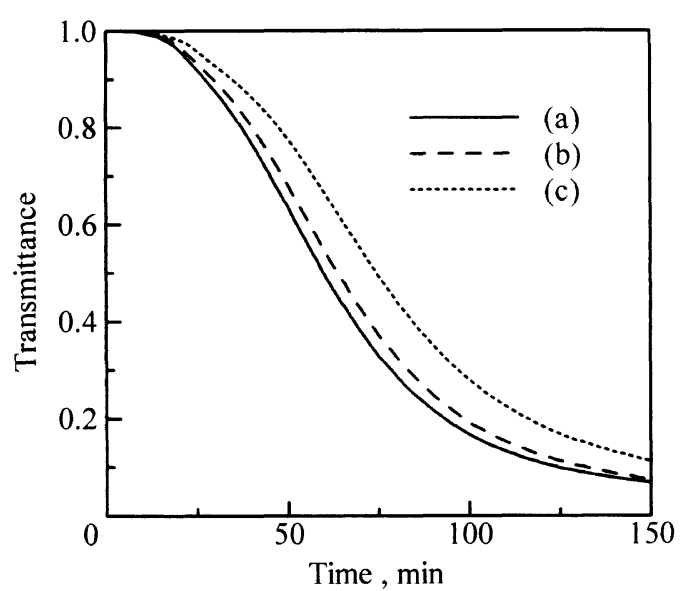

Figure 3. Transmittance curve at $550 \mathrm{~nm}$ for pseudo-polyrotaxane formation between $\alpha$-CD and Az-PEG1 (a), Az-PEG4 (b), and Az-PEG5 (c) in water at $25^{\circ} \mathrm{C}$.

rotaxane with $\alpha$-CDs and the pseudo-polyrotaxane aggregates in the aqueous solution. ${ }^{18}$ The rate of transmittance change was obtained from the slope of the transmittance curve in the linear region. The rate of transmittance change was increased with the Az-PEG concentration (Az-PEG1-3 in Table I ). The rate of transmittance change was fitted by the following logarithmic equation:

$$
r=a \log [\mathrm{Az}-\mathrm{PEG}]+b
$$

where $r$ and [Az-PEG] denote the rate of transmittance change and Az-PEG concentration, respectively. Equation 1 is the most suitable one to represent a relationship between the rate of transmittance change and Az-PEG concentration. From the least-squares fit, $a$ and $b$ of eq 1 were calculated to be 3.03 and 0.86 , respectively. Thus, the rate of transmittance change was in proportion to the logarithm of Az-PEG concentration when the terminal $\mathrm{Az}$ groups of $\mathrm{Az}-\mathrm{PEG}$ were isomerized to the trans isomer.

\section{Effect of cis-Isomerization on the pseudo-Polyrotaxane Formation}

In the case of Az-PEG4 and 5 in Table I, some of the terminal Az groups were photoisomerized by UVirradiation. In spite of the same Az-PEG concentration, the rate of transmittance change decreased with an increase in the cis isomer content (Figure 3). This result suggests that the cis-isomerized $\mathrm{Az}$ moiety hinders pseudo-polyrotaxane formation between $\alpha$-CDs and PEG. The influence of cis $\rightarrow$ trans thermal isomerization on the transmittance change during this experiment is negligible, because the content of thermally isomerized Az-PEG calculated from $k\left(=4.8 \times 10^{-6} \mathrm{~s}^{-1}\right)$ is below $2 \%$ during $1 \mathrm{~h}$.

It is considered that Az-PEG 4 and 5 solutions contain the following two types of Az-PEG derivatives; one is Az-PEG in which only one of both terminals or none of those was cis-isomerized ( 1 or 0 cis Az-PEG) and the other is Az-PEG in which both terminals were cis-isomerized (2cisAz-PEG). In order to clarify the effect of cisisomerization on the pseudo-polyrotaxane formation quantitatively, it is assumed that Az-PEG having two cis-isomerized $\mathrm{Az}$ groups does not participate in the pseudo-polyrotaxane formation, i.e., 1 or 0 cis Az-PEG forms the pseudo-polyrotaxane but 2 cis Az-PEG does not. The content of the 1,0 cis Az-PEG $\left(C_{\mathrm{RX}}\right)$ can be estimated by a following equation:

$$
C_{\mathrm{RX}}=1-\left(\frac{f}{2}\right)^{2} \times\{c i s\}^{2}
$$

where $f$ and $\{c i s\}$ denote the functionality of Az-PEG and the content of cis isomer, respectively. The second term in the right-hand side of the eq 2 represents the content of 2cisAz-PEG. Az-PEG concentration participating in the pseudo-polyrotaxane formation ([AzPEG $]_{\mathrm{RX}}$ ) can be obtained from the product between total Az-PEG concentration ([Az-PEG]) and $C_{\mathrm{RX}}$ :

$$
[\mathrm{Az}-\mathrm{PEG}]_{\mathrm{RX}}=C_{\mathrm{RX}} \times[\mathrm{Az}-\mathrm{PEG}]
$$

As for Az-PEG1-3, [Az-PEG $]_{\mathrm{RX}}$ is the same as [Az-PEG] because all-trans Az-PEG can participate in the pseudo-polyrotaxane formation $\left(C_{\mathrm{RX}}=1\right)$. Thus, the logarithmic relationship could be obtained between $[\mathrm{Az}-\mathrm{PEG}]_{\mathrm{RX}}$ and the rate of transmittance change $(r)$ as mentioned in the previous subsection:

$$
r=a \log [\mathrm{Az}-\mathrm{PEG}]_{\mathrm{RX}}+b
$$

Figure 4 shows a semi-log plot of the rate of transmittance change vs. [Az-PEG $]_{\mathrm{Rx}}$ including Az-PEG4 and 5. This figure shows that eq 4 is available for Az-PEG4 and 5. From the least-square fit including Az-PEG4 and 5, a and $b$ of eq 4 were calculated to be 2.94 and 0.87 , respectively. These values of $a$ and $b$ were the similar ones for eq $1(a=3.03, b=0.86)$. If Az-PEG having two cis-isomerized $\mathrm{Az}$ groups participated in the pseudopolyrotaxane formation, the points of Az-PEG4 and Az-PEG5 in Figure 4 would lie below the point of Az-PEG1. This is because these experiments were carried out on the same Az-PEG concentration as Az-PEG1. From this consideration, it is suggested that Az-PEG having two cis-isomerized Az groups does not participate in the pseudo-polyrotaxane formation. In other words, this result suggests that 2 cis Az-PEG actually does not participate in the pseudo-polyrotaxane formation. 


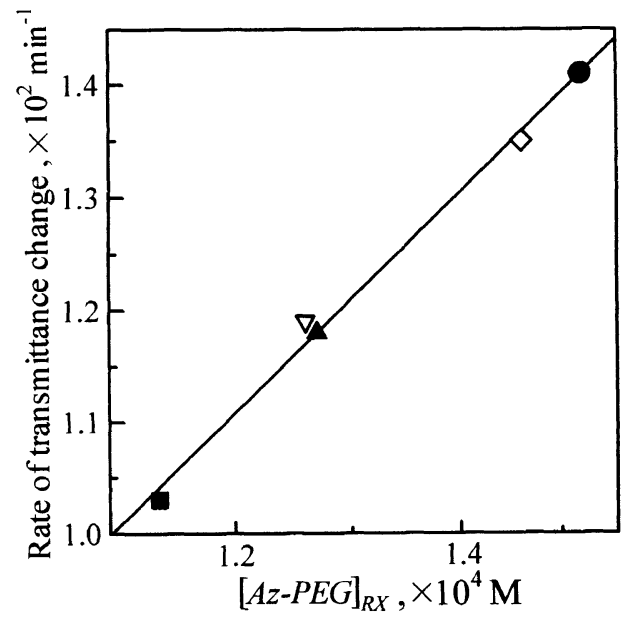

Figure 4. Relationship between the concentration of Az-PEG participating in the pseudo-polyrotaxane formation and the rate of transmittance change; Az-PEG1 (O), Az-PEG2 (A), Az-PEG3 (⿴囗十), Az-PEG4 ( $\diamond)$, Az-PEG5 $(\nabla)$.

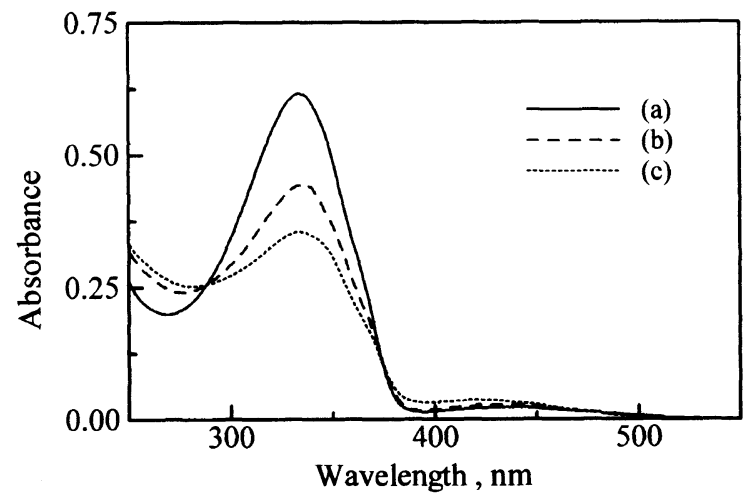

Figure 5. UV-Vis spectra of (a) Az-PEG6 and $\alpha$-CD solution just after mixing, (b) Az-PEG7 and $\alpha$-CD solution just after mixing, and (c) the supernatant of Az-PEG7 after the centrifugation.

\section{The Effect of Isomerization of the Terminal-Az Groups on pseudo-Polyrotaxane Formation}

The rate of transmittance change observed in this study is considered to indicate the sum of the aggregation behavior contributed by the pseudo-polyrotaxane containing various kinds of Az-PEG derivatives. For instance, the pseudo-polyrotaxane containing 1cisAzPEG might aggregate much slower than that without isomerization. Then it may be necessary to confirm the result derived from the transmittance measurement by an alternative method.

It was reported that $\alpha$-CD forms pseudo-polyrotaxane with PEG in aqueous media, and that the pseudopolyrotaxane can be obtained in crystalline state. ${ }^{10}$ In order to analyze the effect of isomerization of terminal- $\mathrm{Az}$ groups on the pseudo-polyrotaxane formation, the content of Az-PEG in the obtained pseudo-polyrotaxane aggregate $\left(\{\mathrm{Az}-\mathrm{PEG}\}_{\text {prx }}\right)$ was determined from ${ }^{1} \mathrm{H} \mathrm{NMR}$ and the supernatant after centrifugation of the aggregate was characterized by the UV-Vis absorption spectrum measurement.

Table II shows the results on the pseudo-polyrotaxane formation between $\alpha-C D$ and Az-PEGs. It was found that the absorbance of Az-PEG solution preparing pseudo-polyrotaxane was drastically decreased after the
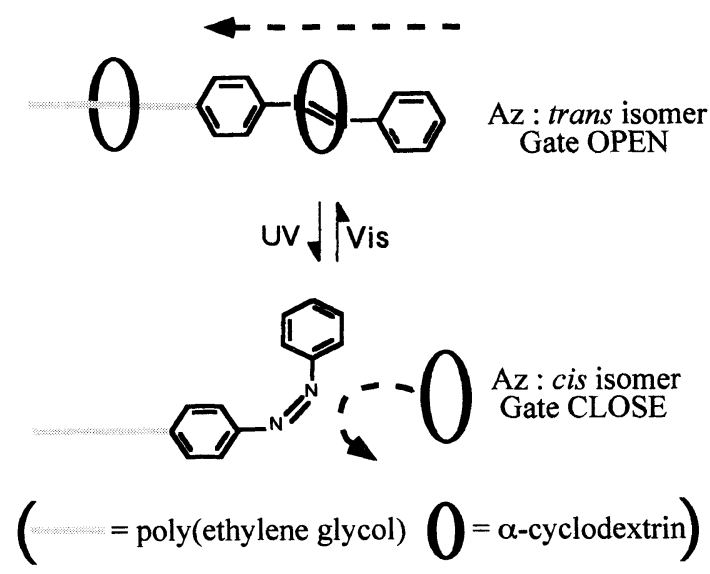

Figure 6. Schematic illustration of stimuli-responsible gate for pseudo-polyrotaxane formation between $\alpha$-CD and PEG.

pseudo-polyrotaxane formation (data not shown). This result indicates the pseudo-polyrotaxane formation between $\alpha$-CD and Az-PEG. From the ${ }^{1} \mathrm{H}$ NMR measurement, 2.3 ethylene glycol units were found per one $\alpha-C D$ molecule, as reported by Harada et al. ${ }^{10}$ Based on this stoichiometry, the content of Az-PEG calculated from the mass of the obtained aggregate after centrifugation $\left(\{\mathrm{Az}-\mathrm{PEG}\}_{\text {prx }}\right.$ ) was determined. As for Az-PEG7 and 8 , it was found that $\{\text { Az-PEG }\}_{\text {prx }}$ was significantly decreased with an increase in the content of cis isomer $(\{c i s\})$. This result supports our suggestion that cis isomer of terminal-Az hinders the formation of pseudo-polyrotaxane.

Since the content of cis isomer in the supernatant was unknown, it is difficult to estimate the content of Az-PEG in the supernatant. To characterize the content of cis isomer in the supernatant, UV-Vis absorption measurement was carried out. Figure 5 shows the UV-Vis spectrum of (a) Az-PEG6 and $\alpha$-CD solution just after mixing, (b) Az-PEG7 and $\alpha-C D$ solution just after mixing, and (c) supernatant of Az-PEG7 after the centrifugation. In this experiment, a diluted Az-PEG solution $\left(5.7 \times 10^{-5} \mathrm{M}\right)$ with $\alpha$-CD saturated solution was used. Since the aggregation of the pseudo-polyrotaxane takes much time in such low Az-PEG concentration, the influence of the aggregation on the UV-Vis absorption was negligible. The absorption maximum of Az-PEG $(325 \mathrm{~nm})$ was shifted to longer wavelength $(330 \mathrm{~nm})$ by adding $\alpha-C D$ solution. In order to compare each absorption spectra, the concentration of Az-PEG was adjusted by using the isosbestic point $(290 \mathrm{~nm})$. It was confirmed that the absorbance at this point was not depend on the cis-isomer content but depend on the Az-PEG concentration (data not shown). From this, the UV-Vis absorption spectra of these three Az-PEG solutions were measured in the same concentration. As shown in (a) and (b) in Figure 5, absorbance at $330 \mathrm{~nm}$ was decreased with an increase in the content of cis isomer. This is because the molar extinction coefficient of cis isomer is much smaller than that of trans isomer in this region. As for the absorption spectrum of (b) and (c), the absorbance of Az-PEG7 at $330 \mathrm{~nm}$ was found to be decreased after the pseudo-polyrotaxane formation. This result indicates that the cis content in the supernatant is higher than that before the pseudo- 
polyrotaxane formation, i.e., the cis-isomerized Az-PEG is concentrated by the pseudo-polyrotaxane formation. This concentration effect was also confirmed in the case of Az-PEG8. Taking these results into account, it is suggested that cis-isomerized Az-PEG hinders the pseudo-polyrotaxane formation. Therefore, it is concluded that terminal Az groups act as a gate to regulate pseudo-polyrotaxane formation between $\alpha$-CDs and PEG (Figure 6).

\section{REFERENCES}

1. Y. Osada and J. Gong, Prog. Polym. Sci., 18, 187 (1993).

2. J.-M. Lehn, in "Supramolecular Chemistry," J.-M. Lehn, Ed., VCH, Weinheim, New York, N.Y., 1995.

3. J. A. Preece and J. F. Stoddart, Nanobiology, 3, 149 (1994)

4. S. I. Stupp, V. LeBonheur, K. Walker, L. S. Li, K. E. Huggins, M. Keser, and A. Amstutz, Science, 276, 384 (1997).

5. H. W. Gibson, M. Gheda, and P. T. Engen, Prog. Polym. Sci., 19, 843 (1994).

6. D. B. Amabilino, F. M. Raymo, and J. F. Stoddart, in "Comprehensive Supramolecular Chemistry," Vol. 9, J.-P.
Saurage and M. W. Hosseni, Ed., Pergamon, Oxford, U.K., 1996, p 85 .

7. G. Wenz, Angew. Chem. Int. Ed. Engl., 33, 803 (1994).

8. A. Harada, and M. Kamachi, in "New Macromolecular Architecture and Functions," M. Kamachi and A. Nakamura, Ed., Springer-Verlag, Berlin, 1996, p 107.

9. A. Harada, M. Okada, J. Li, and M. Kamachi, Macromolecules, 28, 8406 (1995)

10. A. Harada, J. Li, and M. Kamachi, Macromolecules, 26, 5698 (1993).

11. A. Harada, J. Li, and M. Kamachi, Nature, 370, 126 (1994).

12. H. W. Gibson, S. Liu, C. Gong, Q. Ji, and E. Joseph, Macromolecules, 30, 3711 (1997).

13. M. Born and H. Ritter, Makromol. Chem., Rapid Commun., 12, 471 (1991).

14. M. Irie, Adv. Polym. Sci., 94, 27 (1990).

15. H. Murakami, A. Kawabuchi, K. Kotoo, M. Kunitake, and N. Nakashima, J. Am. Chem. Soc., 119, 7605 (1997).

16. B. Gallot, M. Fafiotte, A. Fissi, and O. Pieroni, Macromol. Rapid. Commun., 17, 493 (1996)

17. A. Fissi, and O. Pieroni, and F. Ciardelli, Biopolymers, 26, 1993 (1987).

18. M. Ceccato, P. L. Nostro, and P. Baglioni, Langmuir, 13, 2436 (1997). 